\title{
NAS Grid Benchmarks: A Tool for Grid Space Exploration
}

\author{
Michael Frumkin \\ NASA Advanced Supercomputing Division \\ M/S T27A-2, NASA Ames Research Center \\ Moffett Field, CA 94035-1000 \\ frumkin@nas . naвa.gov
}

\author{
Rob F. Van der Wijngaart \\ Computer Sciences Corporation \\ M/S T27A-1, NASA Ames Research Center \\ Moffett Field, CA 94035-1000 \\ wijngaar@nas.nasa.gov
}

\begin{abstract}
We present a henchmark suite for compurational Grids. It is based on the NAS Parallel Benchmarks (NPB) and is called NAS Grid Benchmark (NGB) in this paper. We present $N G B$ as a data flow graph encapsulating an instance of an NPB code in each graph node, which communicates with other nodes by sending/receiving initialization data These nodes may be mapped to the same or different Grid machines. Like NPB, NGB specifies several different classes (problem sizes). NGB also specifies the generic Grid services sufficient for running the suite. The implementor has the freedom to choose any Grid environment. We describe a reference implementation in Java, and present some scenarios for using $N G B$.
\end{abstract}

\section{Introduction}

The NAS Parallel Benchmarks (NPB) were designed to provide an objective measure of the capabilities of hardware and software systems to solve computationally intensive Computational Fluid Dynamics problems relevant to NASA. They are considered representative of an important segment of high performance scientific computing. At the time of NPB's inception in $15 y_{1}$ there were no accepted standards for progtamming parallel computers, and there was great diversity in hardware systems. It was deemed that any specific benchmark implementation would be unfairly biased towards a certain system configuration or programming paradigm. Hence, the first version of NPB, referred to as NPB1 [1], consisted of a paper-and-pencil specification, with virtually all aspects of the implementation left to the user. A reference implementation, mostly in Fortuan, was provided for the convenience of users, but no claims were made about algorithmic efficiency or appropriatcncss for any particular system.

Despite its apparent lack of concreteness, NPB 1 was em- braced by vendors and researchers, and served as a fruitful lesting ground for programming tools and compiler optimizations. Once a cerlain convergence in programming paradigms was reached, MPI (Message Passing Interface) being the first generally accepted standard, a source code implementation, termed NYB2, was released [2], which became the de facto yardstick for toting (parallelizing) comapilers and tools. Recently, NPB2 was extended to other programming paradigms: OpenMP, HPP (High Performance Fortran) and Java $[5,6,7]$.

Computational and data Grids $[8,16]$ are currently in a state of development comparable to that of high performance computers at the end of the 1980s. Several prototype Grid tools exist (e.g. Globus [9], Legion [18], CORBA [3], Sun Grid Engine [17], Condor [11]), whose relative merits are not well understood. Horo we present a new benchmark suite, the NAS Grid Benchmarks (NGB), which aims to provide an exploration tool to Grid developers and users, similar to those which NPB provided to high-performance computing developers and users. NGB addresses one of the most salient features of Grid computing, namely the ability to execute distributed, communicating processes.

Our pencil-and-paper specification will serve as a uniform tool for testing functionality and efficiency of Grid environments, giving Grid developers a clear target. However, Grids add new diruensiuns to the computational process that require us to reconsider the traditional approach to benchmarking, which focuses on testing the limits of a single computer system. For example, the Grid is heterogeneous, involving different computers, networks, fle servers, and execution environments. Moreover, Grid configurations are dynamic, deprecating common metrics like peak performance and throughpue, and highlighting others, such as latency and failure localization.

Keeping these features in aind, we propose job menaround time as the most important quantitalive metric for henchmarking the Grid. Derivative metrics, such as aggregate cost of tesources (disk space, CPU time, memory, 
1 
network bandwidth) used to complete the benchmark, are currently considered too poorly defined to have utility outside the benchmarker's own organization. NGB will provide a more detailed report on the performance of Grid components, including time to communicate data between any two benchmark tasks executed on (potentially different) platforms, and wall clock time for each task. But since NGB dues not specify how many or which resources to employ. the detailed report is considered diagnostic in narure.

While it may he desirable to determine Grid efficiency in terms of performance achieved divided by resources used, this is an unattainable goal in the near future, since it requires normallzation of resources (Grid fungibility). But it may well be useful for a Grid doveloper to examine increase, or degradation, of NGB performance if a single resoutce within a baseline configuration is varied, such as number of processors in a parallel computation, or number of nctwork hops traversed in a distributed computation.

We expect that initial quantitative NGB performance, even on nominally identical Grid configurations, will show poor repeatability, because performance is often influenced by (currently) unpredictably fiuctuating loads on certain Grid components. As Grid computing matures, more emphasis will be placed on reliability and quality of service. This will be achieved by more explicit control over Grid resources that will allow the user to allocate processing power, memory, network band width, and disk space. NGB can help determine how well claimed resources are actually heing delivered. However, such resource control will not be part of the NGB specification, just as the ability temporarily to suppress time shariag on the processors executing an NPB code was not part of the NPB specification.

We provide an NGB reference implementation in Javaa prototype of which is described in this paper-for the convenience of Grid developers. This implementation allows. us to demonstrate some NGB usage scenarios, and to show sorne interesting effects that users may encounter when benchmarking a Grid.

\section{Grid Benchmarking Requirements}

Grids provide informational and computational environments that deliver new qualities and services to users. Grid benchmarks should explore these new parts of the Grid space and should avoid mapping previously covered cornputing areas. The Grid's informational component should provide fast, well focused and detailed search, storage, and retrieval of data, reliable collaboration between users, access to remote instruments, etc. These services, which are relatively application specific, require their own set of benchmarks, to bc doveloped elsewhero. Wo concentrate, instead, on the computational aspect of the Grid environment, i.e. services used primarily for running computation- ally demanding jobs and processing substantiol dnta sets. Hence, useful benchmark metrics are tumaround time and throughput. Turaround time is time between starting a job and obtaining the resulting data. Throughput is submission volume possible without affecting the turnaround time.

For benchmarking throughput, the Grid has to be stressed to the limit of one of its resources. This can be done, for example, by submitting a large number of instances of the same job with different initial data (parameter study). A throughput benchmark must be intrusive, consuming at least one of the (7rid resources completely. We consider intrusion undesirable for a Grid benchmark, one of whose important functions is to provide continual information on the health of the Grid enviromment. Hence, we will not measure throughput.

NGB tasks are defined in terms of data flow graphs (see Sections 3 and 4 ), whose nodes and arcs represent computations and communications, respectively. An NGB measurement of Grid performance is a report on the execution trace, which Inclurles durations of execution of each node and of transmission along each arc. Summation of such durations along a critical path in the instantiation of the NGB data flow graph (Section 4) on the Grid gives turnaround time, which is al6o reported.

NGB should be representative of tasks typically executed on the Grid, but should also specify well-defined. measurable quantities of work. This precludes, for example, any interactive or nondoterministic processes. A benchmark performance figure is meaningless if the results are wrong, so it is important that a reliable verification test be provided.

NGB should also measure, to some extent, the data transfer capabilities of the communication network, particularly latency and bandwidth. Latoncies are aubomatically included in the turnaround time if communicating tasks of the NGB are executed on a nontrivial subset of the Grid. In order to test batudwidth the benchmark has to send sizeable data volumes. Suilable candidate applicadions for Grid computing are relatively coarse-grained, as latencies between geographically separated Grid platfoxms are often large. This characteristic should be reflected in NGB.

NGB should contain little initialization data. This is a consequence of the paper-and-pencil specification of NGB, since any initialization data should be described in just a few pages of typed toxt. While this requirement could be waived, in principle, once a down-loadable NGB source code implementation is provided, we consider a fairly small source code distribution size desirable.

Grid resources are typically controlled by a certification mechanism that allows a legitimate user to access resources anywhere on the Grid. This means that the user's ability to run jobs is independent of haviog accounts on the individual machinos constituting the Grid. NGB should test the availability of this and other basic Grid services. Paraphrasing 
the pomenclature of $[8$, p. 37], we consider the following set of basic sorvices necessary and sufficient for running jobs on the Grid: authenticate, create task, communicate. The NGB uses these three services and can be executed in any Grid implementation that provides them.

NGB will neither measure nor require security and fault tolerance, even though these are crucial ingredients of a successful Grid. They are very hard to quantify. However, it is envisioned that they will become un infuromal part of NGB performance reporting. For example, turning security on/off may affect NGB turnaround time, and NGB failure rates can indicate Grid reliability. Similarly, while it is vital to know which resources were involved in a certain performance result, we have no way of formalizing their characterization at present. We envision that resource usage will also become an informal part of NGB performance reporting, for studying the trade-off between turnaround time and consumed resources, and crentually for pricing Grid resources.

\section{NGB Design}

To meet the Grid benchmarking requirements we base NGB on two fundamental concepts, listed below.

Basic set of Grid services. Services necessary and sufficient for running NGB are authenticate, create tack, and communicate (see Section 2). They do not assume any particular implementation, hence no Globus, Legion, Condor, CORBA, Grid Engine or other middleware bindings are specified.

Modular structure, open archirecture. An NGB of a particular class (problem size) is specified by a data flow graph encapsulating NGB tasks (NPB codes) and communications between these tasks. Each node and urc of the graph is endowed with a vexification test to determine correctness of its action. This makes it easy to change parameters, organize the bencbmarks into classes, and diversify the tasks in the future. Users can augment or customize the NGB with theit own favorite applications, as long as proper verification tests are supplied, but only the NAS NGB version allows comparisons among different users. The decision to use NYB codes in the baseline NGB, specifically BT, SP, LU, MG, and FT, is motivated as follows.

- The NPB codes are well-studied, well-understood. portable, and widely accepted as scientific benchomark codes.

- Solid verification procedures for NPB already exist.

- The NPB codes require no interaction, and no data fles to start, in principle (but see next item).

- The NPB codes produce sizeable arrays representing solutions on discretization meshes. These can be used as input for any of the other codes, since each is based on structured discretization meshes covering the same physical domain. Hence, it is fairly straightforward to construct siouple but plausible dependency graphs representing sets of interrelated tanks on the Grid, with significant data flows (solution arrays) connecting them.

- The grapularity of the benchmark can tasily bc controlled by varying the number of iterations carried out by each NPB code.

- The NPB codes perform operations that can sensibly symbolize sciendific computation (flow solvers: SP, BT, LUT), post-processing (data smoother: MG), and visualization (spectral unalysis: FT). Collections of such tasks are deemed suitable candidates for Grid computing.

- Good parallel versions of all NPB codcs cxist, which exables balancing the load of complicated Grid tasks by assigning different amounts of computational resources to different subtasks.

\section{NGB Data Flow Graph}

A $\Omega$ instance of NGB comprises a collection of NPB . codes, each of which solves a problem defined on a fixed, logically cubic discretization mesh. Each NPB code (BT, SP, LIJ, MG, or FT) is specified by class (mesh size), number of iterations, provider(s) of the input data, and consumer(s) of solution values. Hence, an instance of NGB is specified by a Data Flow Graph (DFG), see Figure 1. The DFG consists of nodes connected by directed arcs. It is constructed such that there is a directed path from any node to the sink node of the graph (indicated by Report in Figure 1). This is necessary to ensure that any failing node will be registered.

All NRB's mesh based problems are defined on the threedimensional unit cube. However, even within the same problem class ( $S, W, A, B$, or $C$ ) there are differeat mesh sizes for the different benchmark codes. Discretization points of meshes of differeal sicte generally do not coincide. In order to use the outpat from one NPB code as input for another, we interpolate the data tri-limearly, and take arithmetic averages of multiple inputs. The methods used by NPB preserve numerical stability under these operations.

DFG Nade. Each node (except Launch and Report) represents a single computational task. It has a set of input and output ares, an interpolator, a solver, and a verifier. If a node is connected to the source nodo (indicated by launch in Figure 1), it receives control directives to initiate the conlputation. Otherwise it receives input data from other nodes through its input arcs, verifies correctness of the input data 


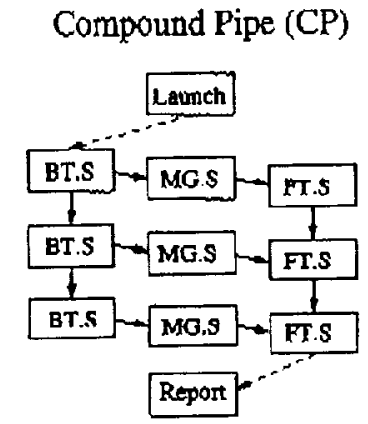

Mixed Bag (MB)
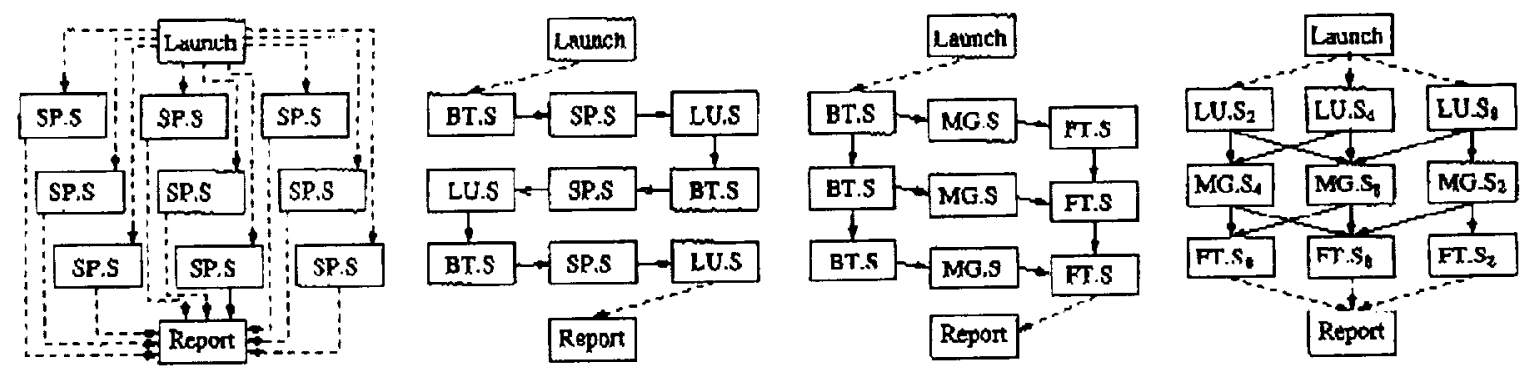

Figure 1. Data flow graphs of NGB, class $S$ (sample size). Solid arrows signify data and control flow. Dashed arrows slgnify control flow only. Subscripts of MB tasks indicate the number of iterations carried out by an NPB code.

and interpolates it to calculate initial conditions. Each node applies the solver to the initial data, and verifies correctness of the computed result. If the node is not connected to the sink node (indicated by Report in Figure 1), it sends the computed solution to all output arcs. Otherwise it sends verification status and timing information to the sink node. Each node contains an instance of NkB2. The implementor is free to attach to the node information on the oomputational resources required for performing its functions. which can be used by a scheduler, see below.

DFG Arc. An arc has tail and head nodes. It represents transmission of data from the tail to the head. The implementor is free to attach to the arc information on the communication resources required for performing its functions, which can be used by a scheduler, see below. Communication along arcs takes place nominally through files (streams, sockets). These may not be cached, but caust be created ancw for each benchmark run. Dashed arcs in Figure 1 connect the nodes Launch and Report to the rest of the graph. They carry no computational data, but are required for control, status, and timing.

NGB Graph Set. NGB employs graphs named Embarrassingly Distributed (ED), Long Pipe (LP), Compound Pipe (CP), and Mixed Bag (MB), as shown in Figure 1. ED represents the important class of Grid applications called parameter studies, which constitute multiple independent nuns of the same program, but with different input parameters. At NASA Ames, flow solvers-symbolized by SPare often used for such studies. LP represents long chains of processes, such as a set of flow computations that are rua one after the other, as is customary when broaking up very long running simulations into series of tasks. CP represents chains of compound processes, like those encountered when visualizing flow solutions as the simulation progresses (see itemized list in Section 3). MB is similar to CP, but now the emphasis is on introducing asymmetry. Differont amounts of data aro transferred between different tasks, and some tasks take longer than others, presenting a tongh job to a scheduling agent that needs to map DFG nodes to resources.

Many important scientific applications consist of iterative processes that are naturally defined in terms of cyclic graphs. Since NGB measures tumaround time, all graphs in the graphs set are noncyclic and finile. They can nonetheless be used to mimic iterative processes, which have as basic building blocks (sets of computational modules whose output can be transformed into input for the same module(s). LP, for example, can be viewed as a partially unrolled cyclic process.

Nodes and arcs are abstract components of the DFG. They are instantiated on a particular set of resources on the Grid, but we do not specify how or where a cextain node or anc of the graph is instantiated. This is part of the beuchorark execution. Executing an arc doos not necessarily imaply accessing a remote machine; multiple nodes may be mapped to the samo platform.

An NGB of a particular class will be submitted for execution on the Grid, presumably to a schedulex. In casc the user does not (want to) specify individual resources for nodes and arcs, the scheduler can make a decision about assigning appropriate Grid resources, depending on the availability of a description of resources requited. This description may include number of floating point operations, memory size, and $\mathrm{I} / \mathrm{O}$ volume.

\section{Reference Implementation}

In keeping with the spirit of the original NPBs, we provide a reference implementation that confums to the rules of the NGB, and can serve as a starting point for developers or users of other Grid environments. It is envisioned that 
standardization of Grid middleware will lead to one or more optimized source code implementations that can be downloaded and run as is (cf. [2])

For the implementation we use the Java platform [10], since it has all the necessary featurcs for creating a rudimentary Grid and NGB application in a compact and portable form. Moreover, a mapping of basic Grid services onto well-established Java constructs is telatively straightforward, and is spocified as follows.

Authentication is provided by the NGB Pad (a Java application written for $N G B$ ), which requires the user to type a user name and password. This authentication acts as a proxy for each NGB task request issued from the NGB Pad. Java support for restricted access to scrviccs provided by the reference implementation is contained in package java.security. We use Java to enable task creation through the object lookup mechanism and aative process creation contained in the $j a v a . r m i$ and $j$ ava.$j n i$ packages, respectively. Communication (along arcs of the graph) is handled by the remote method invocation mechanism and file $\mathrm{V} / \mathrm{O}$, contained in the java . rmi and java. jo packages, respectively.

The reference implementation involves two levels: the Grid level, which provides the basic Grid services, and the application level, which makes use of the services to implement the NGB tasks. NGB developers are expected only to implement the application level.

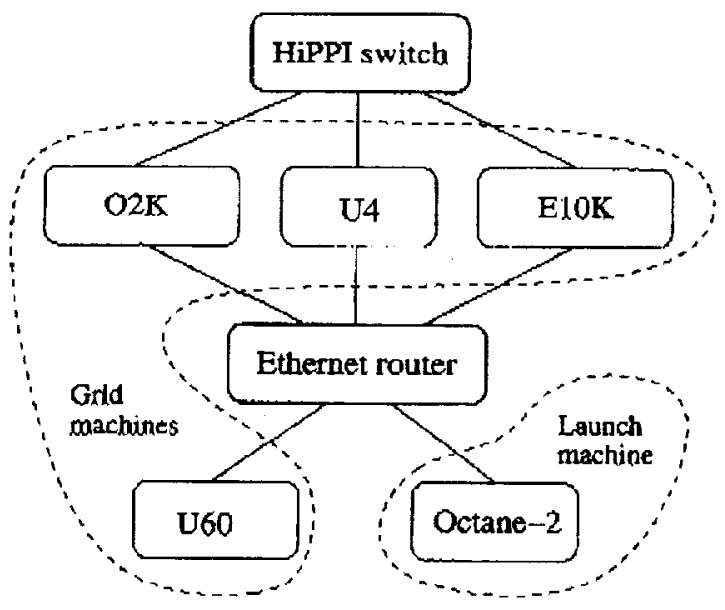

Figure 2. Network topology of machines used in the experiments.

Grid level. Our rudimentary Grid environment services the user's requests for nuning benchmarks and for repoiting results. Installation of the environment by a Grid administrator involves the following steps:
- Install Java registry and bind services to the registry on all Gid machines (executing NPB codes). At present, the services are specific to NGB, but they can be made completely generic. Once they are, the Grid administrator no longer needs to be involved in installing user codes.

- Specify the sccurity policy and eligible users.

- Install NGB Pads on Launch machines-from which NGB execution is initiated-to provide authentication and access to Grid services. At present, authentication and the application interface are integrated, which limits the generality of our rudimentary Grid enviromment.

Application level. The application level uses the basic Grid services to implement the NGB. Launch machines are created by the installation of the NGB Pad. The Pad, which can run on any machine that is able to access Grid machines via the HTTP protocol, is a graphical user interface (Figure 3) that encapsulates the basic Gid services. It reads a textual description of the DFG, turns it iuto a graph abjech, subwits it for execution on the Grid specified within the DFG, and collects and displays the benchmark perfoxmance report. Currently, nodes of the DFG have to bo mapped onto the Grid explicitly. The mapping includes the name of a Grid machine, number of processors used, and a programming paradigm (MPI, Java, HPF, OpenMP). We use an explicit mapping, since a connection with a Grid scheduler (resource broker) has not boen implemented at the time of writing. In return, the uscr gcts a bencbmark performance report containing turnaround time and verification status for the whole benchmark, and for each node in the DFG.

Benchroark installation and execution involves the following steps:

- Compile and install NPBs on Grid machines (currently requires Grid administrator privileges and is therefore carried out in the installation step at the Grid level).

- Create DFG (in textual format).

- Invoke NGB Pad.

- Identify self as NC.B Grid user.

- Submit DFG for execution on the Grid.

- Obtain benchmark repoit.

Experimental Setup. We installed the NGB services on a compact Grid comprised of 4 machines at NAS: a 24 procossor SGI Origin 2000 (O2K), a 16-processor SUN E10000 (E10K), a 4-processor SUN Ultra-4 (U4), and a 2-processor SUN Ultra-60 (U60). The machines were connected by fast, switched Ethernet-supplemented with some HifPI connections-with a typical round-trip time of $0.5 \mathrm{~ms}$, see Figure 2. Only one processor was used on each of the machines. The NGB Pad ran on a single processor 


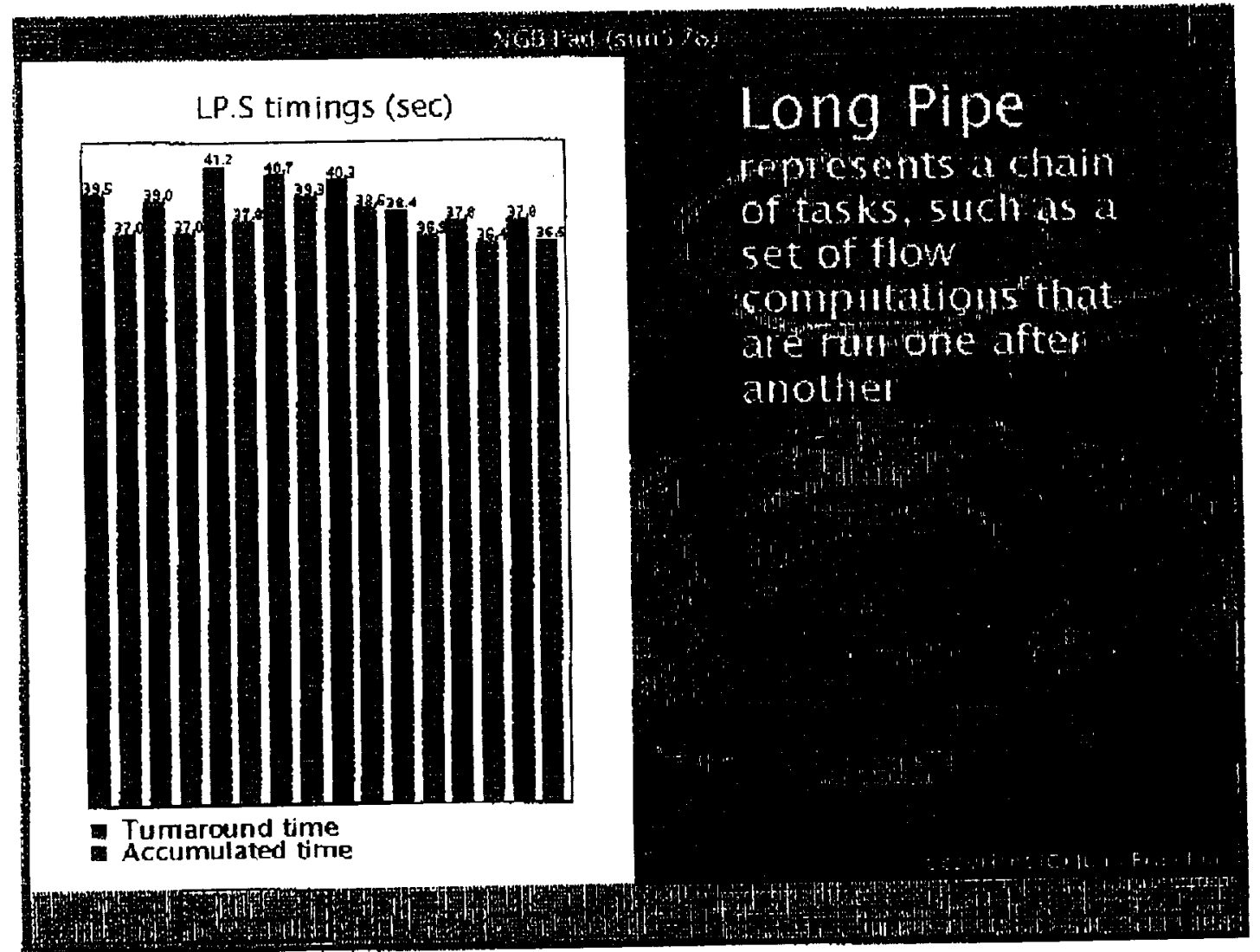

Figure 3. Prototype NGB Pad Graphical User Interface. Background artwork reproduced with permission of the artist, Jusia Frumkin.

SGI Octane-2 by a user who did not bave accounts on two of the four Grid machines.

Experimental Results. We executed the LP.S benchraark (Figure 1), mapped onto the ring U60-O2K-E10K-U4, in three modes, illustrated in Pigure 4. Here each "wave" constitutes a snapshot of an instance of LP.S, which has at most one executing task (sharp peak) at any one time. Figures $4 \mathrm{~b}$ and $4 \mathrm{c}$ each show snapshots of two instances of L.S.S, mapped onto the ring of Grid machines in the sarde (b) and opposite $(0)$ directions, respectively. The co-directed waves were started at different times, so that they never $\alpha$ cupied the same machine simultaneously. We measured the turnaround time of LP.S and accumulated CPU time for all nodes of I.PS for a number of runs during a single day. Results shown in Figure 5 provide quantitative insight into Grid overhead and timing consistency.

The graphs show that the compact Grid-whose machines were not running in dedicated mode for our experiments-exhibited the timing consistency of a single processor in a multiuser mode, keeping variation of the turnaround time within $10 \%$. We define Grid overhead as the difference between total turnaround time-which includes time spent in communications-and accumulated CPU time within the nodes of a node critical path. A node critical path is defined as the critical path of the instantiation of the DPG when iguoring all communication and synchronization times. For the LP benchmark the Grid overhead is simply the difference between turaaround time and accumulated CPU time. It follows from Figure 5 that the Grid overhead was below $5 \%$ percent for all cases, indicating that the communications did not dominute the benchmark executions, and that the Java server overhead was acceptable. Apparently, the load experienced by the NAS machines was steady during the period of testing.

We also made the following observations in our oxpeniments, see Figure 5. Solitary waves (left) exhibited a steady 
a)

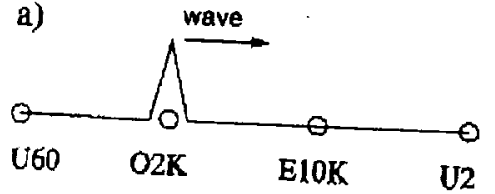

b)

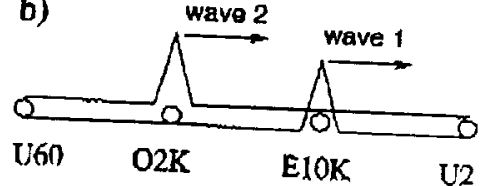

c)

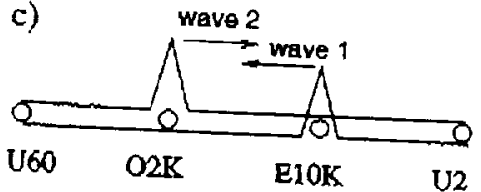

Figure 4. Solltary and binary waves in Grid saco. waves travel in the eame (b) and opposite (c) directions, respectively.
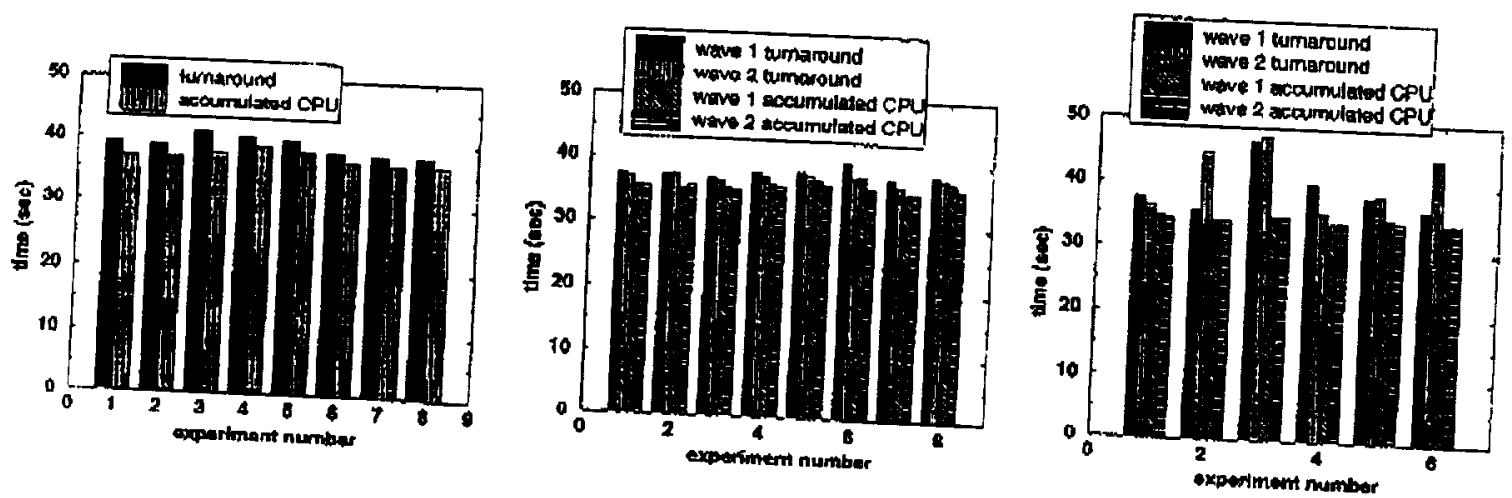

Figure 5. One-day sampling of compact Grid performance using LP.S.

behavior in Grid space. Co-directed binary waves (middle) exhibited behavior similar to that of solitary waves. The second wave, traveling in the "wake" of the first, had a binary waves (right) reverhead. Collisions of antidirected overhead. (right) resulted in occasional increase of Grid

While we should resist drawing deep conclusions from this simple experiment, it illustrates how NGB may serve to plications on the Grid.

\section{Related Work and Conclusions}

A number of projects intend to probe the quality of Grid environments, for example, the Globus Heart Beat Monitor [15] (part of the Glabus [9] metacomputing toolkit), the Network Weather Service [14], and the simulation projects

The Hearr [4], MicroGrid [12], and Bricks [13]. and periodic heartbears to be allows a process to be tracked but it provides no performance statistics. Network Weather Service is statistics. The goal of the of dynamically changice is to provide accurate forecasts a distributed set of metacerformance characteristics from aim to provide a metacomputing resources. It does not mance on different hardwared set of tasks whose perforpared meaningfully.
The WARMstones projoct does plan to provide a touchstone for realistic comparisons between scheduling algoby simulating the systemibuted systems, bur it will do so performance results from under considcration and desiving MicroGrid projects from the simulation. The Bricks and cally targets distributed similar goals. MicroGrid specifiof Clobus [9].

NGB complements the above cfforts by ptoviding standardized tests that are not tied to any particular Grid middieware, but that nonetheless can be execured within a real Grid environment. This allows NGB to be used for regrosent Gests of Grid services, and to compare between different Grid environments. A full specification and referenco isoplementation will be released shortly.

NASA High Perfors. This work was supported by the Program, RTOP \#725- Computing and Communications bers of the Algorithms. NASA Ames' NAS division for Architectures group in and to Sandy Johan for reviewing constructive suggestions,

\section{References}

[1] D.H. Bailey, J. Barton, T. Lasinski, and H. Simon (Eds.). The NAS Parallel Benchmarks. NAS Technical 
Report RNR-91-002, NASA Ameo Research Centex, Moffett Field, CA, 1991.

[2] D.H. Bailey, T. Harris, W.C. Saphir, R.F. Yan der Wijngaart, A.C. Woo, M. Yarrow. The NAS Parallel Benchmarks 2.0. NAS Technical Report NAS-95-020, NASA Ames Research Center, Moffett Field, CA. 1995

[3] R. Ben-Naten. CORBA: A Guide to Common Object Request Broker Architecture. McGraw-Hill, New York, 1995.

[4] S.J. Chapin, WARMstones: Benchmarking Wide-Area Resource Management Schedulers. Draft white paper, Syracuse Univorsity, http://www,hpde.syr.edu/chapin/curxentproj.html.

[5] H. Jin, M. Frumkin, 3. Yon. The OpenMP Implemensation of NAS Parallel Benchmarks and Its Pefor. mance. NAS Technical Report NAS-99-011, NASA Ames Research Center, Moffett Field, CA, 1999.

[6] M. Frumkin, H. Jin, J. Yan. Implementation of NAS Parallel Benctmarks in High Performance Fortran. Proc. Intemational Parallel Processing Symposium, 1999, hatp://ipdps eece.unm edu.

[7] M. Frumkin, M. Schultz, H. Jia, J. Yan Implementation of NAS Parallel Benchmarks in Java. Presented at a Poster session at ACM 2000 Java Grande Conference, 2000.

[8] The Grid. Blueprint for a New Computing Infrastructure. I. Foster, C. Kesselman, Eds-, Morgan Kaufmann Publishers Inc., San Francisco, CA, 1999.

[9] I. Foster, C. Kesselman. Globus: A Metacomputing Infrastructure Toolkit. Int. J. Supercomputer Applications, 11(2):115-128, 1997, http://www.globus.org.

[10] C.S. Horstmann, G. Cornell. Core Java 2, Volume 2: Advanced Features. 4th edition, Prentice Hall, 1999, see also http://java.sun.com/j2se/1.3/docs.

[11] M. livny, J. Basney, R. Raman, T. Tannenbaum. Mechanisms for High Throughput Compuring. SPEEDUP Journal, Vol. 11(1), 1997 , http://www.cs.wisc.edu/condor/.

[12] H.J. Song, X. Liu, D. Jakobsen, R. Bhagwan, X. Zhang, K. Taura, A. Chicn. The MicroGrid: a Scientific Tool for Modeling Computational Grids. Proc. Supercomputing 2000, Dallas, TX, 2000.

[13] A. Takefusa, S. Matsuoka, H. Nakada, K. Aida, U. Nagashima. Overview of a Performance Evaluation
System for Global Computing Scheduling Algorithms. Proc. High-Performance and Distributed Computing 8, pp. $97-104,1999$.

[14] R. Wolski, N.T. Spring. J. Hayes. The Nerwork Weather Service: A Distributed Resource Performance Forecasting Service for Metacomputing. J. Future Generation Computing Systems, 1999, also UCSD Technical Report Number TR-CS98-599, 1998, http://nws-npaci.edu/NWS/.

[15] Globus Heartbeat Montitor. htt://www.globus.org/hbm/.

[16] NASA Information Power Grid. http://www.nas.nasa.gov/TPG.

[17] Codine 5.2 Manual, Revision A. Sun Microsystems, Inc., Palo Alto, CA, September 2000, http://www.sun.com/gridware.

[18] Legion 1.6, Developer Mankal. The Legion Research Group, Dept Computer Science. U. Virginia. Charlottesville, VA, 1999, http//hegion.virginia.edu. 
